\title{
A VARIATION ON THE STONE-WEIERSTRASS THEOREM
}

\author{
R. I. JEWETT
}

If $X$ is a set, let $I^{\boldsymbol{x}}$ be all functions from $X$ into the unit interval $I$. Note that if $f$ and $g$ are in $I^{\boldsymbol{x}}$ then so are $1-f$ and $f g$. Such a collection of functions is said to have property $\mathrm{V}$. That is, $F$ has property $\mathrm{V}$ in case
(i) $F \subset I^{x}$ for some set $X$,
(ii) $f \in F$ implies $1-f \in F$,
(iii) $f, g \in F$ implies $f g \in F$.

Giving $I^{X}$ the topology of uniform convergence, we have that the closure of a set with property $\mathrm{V}$ has property $\mathrm{V}$, as does the intersection of such sets. Thus every subset of $I^{\boldsymbol{x}}$ is contained in a smallest set with property $\mathrm{V}$, and in a smallest closed set with property $\mathrm{V}$. If $X$ is a topological space then the set $D(X)$ of all continuous functions from $X$ into $I$ is closed and has property $V$. The idea of considering such collections of functions comes from a statement of von Neumann in [1]. Essentially, he claims without proof what we give here as a corollary to Theorem 2. I am indebted to Dr. R. S. Pierce for bringing the problem to my attention.

Definition. If $n$ is a positive integer, let $P_{n}$ be the smallest subset of $D\left(I^{n}\right)$ that has property $\mathrm{V}$ and contains the $n$ projections.

Lemma 1. Let $F$ have property $\mathrm{V}, p \in P_{n}$, and $f_{k} \in F$ for $k=1,2$, $\cdots, n$. Then the function $f$ defined by

$$
f(x)=p\left(f_{1}(x), f_{2}(x), \cdots, f_{n}(x)\right)
$$

is in $F$.

Proof. Let $Q$ be the set of all $q \in D\left(I^{n}\right)$ for which $q\left(f_{1}(x), f_{2}(x)\right.$, $\left.\cdots, f_{n}(x)\right)$ is in $F$. Then $Q$ has property $\mathrm{V}$ and contains the $n$ projections. So $Q$ contains $P_{n}$.

Lemma 2. If $a<b$ and $\epsilon>0$, then there exists $p \in P_{1}$ such that

$$
\begin{array}{ll}
p>1-\epsilon & \text { in }[0, a], \\
p<\epsilon \quad \text { in }[b, 1] .
\end{array}
$$

We set $[0, a]=\varnothing$ if $a<0$ and $[b, 1]=\varnothing$ if $b>1$.

Proof. Since for a sufficiently large integer $k, x^{k}(1-x)^{k}<\epsilon$ and

Received by the editors August 2, 1962. 
$1-x^{k}(1-x)^{k}>1-\epsilon$ for all $x$ in $I$, we can assume that $0 \leqq a$ and $b \leqq 1$. Also, since there exist $a^{\prime}, b^{\prime}$ such that $a<a^{\prime}<b^{\prime}<b$, we can assume that $0<a<b<1$. Now our solution will be of the form $p(x)=\left(1-x^{m}\right)^{n}$. Pick $r$ such that $\left(\frac{3}{4}\right) r<\epsilon$. Pick $m, s$ such that

$$
\left(\frac{3}{4}\right) \frac{1}{b^{m}}<s<\frac{1}{b^{m}}<\left(\frac{\epsilon}{r}\right) \frac{1}{a^{m}} .
$$

Let $n=r s$ and note that $n a^{m}<\epsilon, \frac{3}{4}<s b^{m}<1$. So

$$
\begin{aligned}
& \left(1-a^{m}\right)^{n}>1-n a^{m}>1-\epsilon, \\
& \left(1-b^{m}\right)^{n}=\left[\left(1-b^{m}\right)^{s}\right]^{r}<\left[1-s b^{m}+\frac{1}{2}\left(s b^{m}\right)^{2}\right]^{r}<\left(\frac{3}{4}\right)^{r}<\epsilon .
\end{aligned}
$$

One can prove by induction that if $0<x<1$ then $(1-x)^{n}<1-n x$ $+\frac{1}{2}(n x)^{2}$.

LEMMA 3. If $a_{k}, b_{k} \in I$ for $k=1,2, \cdots, n$ then

$$
\left|\prod_{1}^{n} a_{k}-\prod_{1}^{n} b_{k}\right| \leqq \sum_{1}^{n}\left|a_{k}-b_{k}\right| \text {. }
$$

Proof. The induction step can be verified as follows. Let $a=a_{1} a_{2} \cdots a_{n-1}$ and $b=b_{1} b_{2} \cdots b_{n-1}$. So $a, b \in I$ and

$$
\begin{aligned}
\left|a a_{n}-b b_{n}\right| & \leqq\left|a a_{n}-b a_{n}\right|+\left|b a_{n}-b b_{n}\right| \\
& \leqq|a-b|+\left|a_{n}-b_{n}\right| .
\end{aligned}
$$

Lemma 4. Let $(a, b) \in I \times I$ and $\epsilon, \delta>0$. Then there exists $p \in P_{2}$ such that

$$
\begin{aligned}
& p(x, y)>1-\epsilon \text { if }(x-a)^{2}+(y-b)^{2} \leqq \delta^{2}, \\
& p(x, y)<\epsilon \text { if }(x-a)^{2}+(y-b)^{2} \geqq(4 \delta)^{2} .
\end{aligned}
$$

Proof. Let the functions $p_{1}, p_{2}, p_{3}, p_{4} \in P_{1}$ correspond by Lemma 2 to $a-2 \delta<a-\delta, a+\delta<a+2 \delta, b-2 \delta<b-\delta, b+\delta<b+2 \delta$ and $\epsilon / 4>0$, respectively. Then let $p$ be given by

$$
p(x, y)=\left[1-p_{1}(x)\right] p_{2}(x)\left[1-p_{3}(y)\right] p_{4}(y) .
$$

Lemma 5. Let $A, B \subset I \times I$ be closed and disjoint. If $\epsilon>0$ and $p \in P_{2}$, then there exists $q \in P_{2}$ such that

$$
\begin{aligned}
& q \geqq p \text { in } I \times I, \\
& q>1-\epsilon \text { in } A, \\
& q<p+\epsilon \text { in } B .
\end{aligned}
$$


Proof. We can assume that $A$ and $B$ are nonvoid. Let $4 \delta$ $=\operatorname{dist}(A, B)$. Then $\delta>0$ and there exist $\left(c_{k}, d_{k}\right) \in A$ for $k=1,2, \cdots, n$ such that the $\delta$-neighborhoods of the $\left(c_{k}, d_{k}\right)$ cover $A$. For each $k$ there exists $q_{k} \in P_{2}$ such that

$$
\begin{aligned}
& q_{k}(x, y)>1-\epsilon / n \text { if }\left(x-c_{k}\right)^{2}+\left(y-d_{k}\right)^{2} \leqq \delta^{2}, \\
& q_{k}(x, y)<\epsilon / n \text { if }\left(x-c_{k}\right)^{2}+\left(y-d_{k}\right)^{2} \geqq(4 \delta)^{2} .
\end{aligned}
$$

Let $q_{0}=\left(1-q_{1}\right)\left(1-q_{2}\right) \cdots\left(1-q_{n}\right)$. It is clear that $q_{0}>1-\epsilon$ in $B$, and $q_{0}<\epsilon / n$ in $A$. Now let $q=1-(1-p) q_{0}$. In $I \times I$ we have $q \geqq 1$ $-(1-p)=p$. In $A$ we have $q \geqq 1-q_{0}>1-\epsilon$. And in $B$ we have $q-p$ $=1-q_{0}+p q_{0}-p=\left(1-q_{0}\right)(1-p)<\epsilon$.

Theorem 1. Let $X$ be a set and $F$ a closed subset of $I^{x}$. If $F$ has property $\mathrm{V}$ then $F$ is a lattice.

Proof. In view of Lemma 1 , it is enough to prove that the functions $(x, y) \rightarrow x \wedge y$ and $(x, y) \rightarrow x \vee y$ of $I \times I$ into $I$ can be uniformly approximated by members of $P_{2}$. Since $x \vee y=1-(1-x) \wedge(1-y)$, it is enough to check $x \wedge y$. Let $0<\epsilon<\frac{1}{4}$ and let $C$ be the set of all $(x, y) \in I \times I$ for which $\epsilon \leqq x \wedge y \leqq 1-\epsilon$. Then $C$ is closed and there exists $m>0$ such that $x^{m} y^{m}<\epsilon$ in $C$. Let $p(x, y)=1-x^{m} y^{m}$. Then $1-\epsilon<p<1$ in $C$. For $k \geqq 0$ let

$$
\begin{aligned}
& A_{k}=\left\{(x, y) \in C \mid p^{k}(x, y) \geqq x \wedge y\right\}, \\
& B_{k}=\left\{(x, y) \in C \mid p^{k}(x, y) \leqq x \wedge y\right\} .
\end{aligned}
$$

Then $A_{1}=C$ and for $k \geqq 0$

$$
A_{k} \supset A_{k+1}, \quad B_{k} \supset C-A_{k}, \quad A_{k+1} \cap B_{k}=\varnothing .
$$

Because the $A_{k}$ have void intersection, there exists $n>2$ such that $A_{n}=\varnothing$. For $k=1,2, \cdots, n$ pick $q_{k} \in P_{2}$ such that $q_{k} \geqq p$ in $I \times I$, $q_{k}>1-\epsilon / n$ in $B_{k-1}$, and $q_{k}<p+\epsilon / n$ in $A_{k}$. Let $q=q_{1} q_{2} \cdots q_{n}$. Now $C=\mathrm{U}_{1}^{n-1}\left(A_{k}-A_{k+1}\right)$. For $k=1,2, \cdots, n-1$ we have in $A_{k}-A_{k+1}$

$$
0 \leqq p^{k}-x \wedge y<p^{k}-p^{k+1}=p^{k}(1-p)<\epsilon .
$$

Also, we have

$$
\begin{aligned}
\left|p^{k}-q\right| & \leqq\left|p^{k}-p^{k+1}\right|+\left|p^{k+1}-q\right|<\epsilon+\left|p^{k+1}-\prod_{1}^{n} q_{j}\right| \\
& \leqq \epsilon+\sum_{1}^{k}\left|p-q_{j}\right|+\left|p-q_{k+1}\right|+\sum_{k+2}^{n}\left|1-q_{j}\right| \\
& <\epsilon+k \frac{\epsilon}{n}+(1-p)+(n-k-1) \frac{\epsilon}{n}<3 \epsilon .
\end{aligned}
$$


Thus in $C,|q-x \wedge y|<4 \epsilon$. Now by Lemma 5 , there exists $q^{\prime} \in P_{\mathbf{2}}$ such that $q^{\prime} \geqq q$ in $I \times I, q^{\prime}>1-\epsilon$ if $x \wedge y \geqq 1-\epsilon$, and $q^{\prime}<q+\epsilon$ if $x \wedge y \leqq 1-2 \epsilon$. Clearly $\left|q^{\prime}-x \wedge y\right|<\sigma \epsilon$ if $x \wedge y \geqq \epsilon$. Similarly, there exists $q^{\prime \prime} \in P_{2}$ such that $q^{\prime \prime} \leqq q^{\prime}$ in $I \times I, q^{\prime \prime}<\epsilon$ if $x \wedge y \leqq \epsilon$, and $q^{\prime \prime}>q^{\prime}-\epsilon$ if $x \wedge y \geqq 2 \epsilon$. So $\left|q^{\prime \prime}-x \wedge y\right|<8 \epsilon$ in all of $I \times I$.

THEOREM 2. Let $X$ be a compact space and $F$ a closed, point-separating subset of $D(X)$ that has property V. If $S$ is the set of points of $X$ taken into the doubleton $\{0,1\}$ by every member of $F$, then $F$ consists of all functions $f \in D(X)$ for which $f(S) \subset\{0,1\}$.

Proof. It is well known [2] that for a compact space $Y$, a closed sublattice of $C(Y)$ contains any continuous function which it approximates at each pair of points. So, let $f \in D(X)$ be such that $f(S) \subset\{0,1\}$, and let $u, v$ be distinct elements of $X$. The case when $X$ has only one element is straightforward.

Suppose $u, v \in S$. Then there exists $g \in F$ such that $g(u) \neq g(v)$, and then one of $g, 1-g, g(1-g), 1-g(1-g)$ duplicates $f$ on $u$ and $v$.

If $u \in S, v \notin S$, then there exists $g \in F$ such that $g(u)=f(u)$ and $g(v) \in(0,1)$. As can be seen from Lemmas 1 and 2, something of the form $1-\left(1-g^{m}\right)^{n}$ will do.

If $u, v \notin S$, then there exist $g_{1}, g_{2}, g_{3} \in F$ such that $g_{1}(v) \leqq g_{1}(u)$ $\in(0,1), g_{2}(u) \leqq g_{2}(v) \in(0,1)$, and $g_{3}(v)<g_{3}(u)$. If we let $h_{1}=g_{1} g_{3}$ and $h_{2}=g_{2}\left(1-g_{3}\right)$, we have $h_{1}(v)<h_{1}(u)<1$ and $h_{2}(u)<h_{2}(v)<1$. Something of the form $f_{2}=\left(1-h_{2}^{r}\right)^{s}$ approximates 1 at $u$ and $f$ at $v$. Something of the form $f_{1}=\left(1-h_{1}^{k}\right)^{m}$ approximates $f$ at $u$ and 1 at $v$. So $f_{1} f_{2}$ approximates $f$ at $u$ and $v$.

COROLlaRy. The smallest closed subset of $D\left(I^{n}\right)$ having property $\mathrm{V}$ and containing the projections and at least one constant $c \in(0,1)$ is $D\left(I^{n}\right)$ itself.

\section{REFERENCES}

1. J. von Neumann, Probabilistic logics and the synthesis of reliable organisms from unreliable components, Automata Studies, pp. 93-94, Princeton Univ. Press, Princeton, N. J., 1956.

2. L. Gillman and M. Jerison, Rings of continuous functions, Van Nostrand, Princeton, N. J., 1960.

UNIVERSITY OF OREGON 